\title{
A Hybrid-based Medical Decision Support System
}

\author{
Ifeanyi Charles Emeto \\ Department of Computer Science, University of \\ Port Harcourt, Choba, Nigeria.
}

\author{
Chidiebere Ugwu \\ Department of Computer Science, University of \\ Port Harcourt, Choba, Nigeria.
}

\begin{abstract}
This paper presents a hybrid model of genetic and back propagation algorithm for the development of a decision support system for kidney stone. The initialization and optimization of the weights connection of ANN for improved performance was done with genetic algorithm. Object oriented analysis and design methodology was used for the design, java programming language was used for the interfaces and database respectively. Experimental results show that the hybrid model classified at higher accuracy $98.84 \%$. This implies that the hybrid system is good for clinical decision making.
\end{abstract}

\section{General Terms}

Machine Learning, Decision Support System.

\section{Keywords}

Artificial Neural Network, Genetic Algorithm, Nephrolithiasis, Neuro-Genetic Model.

\section{INTRODUCTION}

Nephrolithiasis is derived from the Greek word Nephros(kidney) lithos (stone) i.e. kidney stone. Kidney stone is a kidney disorder; it is made up by the formation of crystalline aggregates ('kidney stone') that can grow anywhere along the urinary tract (or lower down in the urinary tract). Kidney stone often causes blood in the urine and pain in the abdomen, flank or groin. Kidney stone are common in western societies; nearly $10 \%$ of Americans will develop a symptomic kidney stone during their life time. It is observed that there are increasing cases of this nature, especially in Nigeria due to the climatic condition.[11] Passing kidney stone can be quite painful, but the stone usually cause no permanent damage.[12] However, stone diseases is among the most painful and prevalent urological disorders. Fortunately most stones pass out of the body without any intervention, there are considerably fewer specialist in the field of Nephrolithiasis, called Urologist, and they are not usually available in all hospitals. Treatment of Kidney stone is a gradual process because it is a chronic kidney condition; it requires regular visits to the hospital to enable the specialist monitor the patient's records which need to be up to date in order to be used in making vital decisions. [13-15][22].

Nephrolithiasis is gradually seen as a systemic disease indicative of an adverse metabolic environment. [1-2] it is a relatively prevalent disease in western civilization [3]. Epidemiological studies documented a relationship of nephrolithiasis with obesity, diabetes mellitus and arterial hypertension. [4-7]. Nephrolithiasis prevalence is greater in warmer climates presumably because higher ambient temperature leads to reduced urine volume and higher concentrations of stone-forming salts.[8] Making the right decision on how to solve a problem can be challenging; this can be even more tasking for a doctor whose decision is pivotal to saving a patient's life.[9] In such cases, the process of arriving at a decision should be based on well refined facts and not instincts. As such the doctor needs to analyze all the data available and arrive at the best decision given the available data.[10] Further efforts have been made to develop hybrid decision support system which is a combination of two or more techniques that ensure the eventual decision reached by the doctor will be devoid of guess.

This paper proposed a hybrid-based diagnostic system as a tool for making vital decision for patients with Nephrolithiasis.

\section{RELATED LITERATURE}

Hybrid computer-based clinical decision support systems (CDSS) are software applications designed to directly aid in clinical decision making in which characteristics of individual patients are matched to a computerized knowledge base for the purpose of generating patient-specific assessments, diagnosing the ailment, guiding the physician in carrying out further verifications, and managing patient's over time.[16]

In [17] a study of hybrid Genetic Algorithm using Artificial Neural Network in Data mining for the Diagnosis of Stroke Disease was presented. In their work, Neural Network and Genetic Algorithm were used to initialize and optimize the connection weights of Artificial Neural Network with the aim of improving the performance of ANN. A data of 150 patients with symptoms of stroke disease was used; the data was analyzed in the dataset to define column parameters and data anomalies. Backward stepwise method was used for input feature selection; the architecture of the Neural Network designed consists of 20 inputs nodes, 10 hidden nodes and 10 output nodes and was trained using back-propagation. The Neuro-Genetic approach, all 20 symptoms are taken into account and the conclusion showed that the new hybrid Neuro-genetic method provided a better accuracy and faster convergence due to complexity of the network. The prediction accuracy is $98.67 \%$.

Abhishek et al. [18] adopted two neural network techniques, Back Propagation Algorithm (BPA), Radial Bias Function $(\mathrm{RBF})$ and one non-linear clarifier support Vector Machine to diagnose kidney stone disease, the BPA, RBF and SVM shared a correctly clarification accuracy of $81.4 \%, 62.2 \%$ and $60.22 \%$ respectively.it was also observed that the back propagation has the lowest error rate 0.2418 than the RBF and SVM, and also the kappa statistic of back propagation is greater than RBF and SVM. The back propagation (BPA) can be seen to significantly improve the conventional classification technique used in the medical field.

Koshal et al. [19] in their work Artificial Neural Networks for Diagnosis of Kidney Stone Disease used three different neural network algorithms which have different architecture and characteristics to diagnose kidney stone disease. Learning vector quantization (LVQ), two layers feed forward perceptron trained with back propagation training algorithm and Radial basis function (RBF) networks were the three neural network used with Waikato Environment for Knowledge Analysis (WEKA) version 3.7.5 as a simulation tool. The authors observed that multilayer perceptron with 
two hidden layers and back propagation algorithm was the best model for diagnosis of kidney stone disease. Its accuracy is $92 \%$ and it correctly classified the 977 instances from 1000 instances.

Quethara [20] in his work evaluated the use of artificial neural network in diagnosis of acute rehritis and heart disease, the feed-forward back propagation neural network with supervised learning was used and the ANN showed significant results in dealing with data represented in symptoms and images, as the \% of correctly classified samples is $99 \%$ using the nephritis disease sample, and $95 \%$ using the heart disease sample, this shows that the ANN is useful in identifying infected person.

Hamada et al [21] applied the hybridization of different machine algorithms (artificial neural networks, fuzzy logic and genetic algorithms) in a complementary manner to overcome the shortfalls of the algorithms in a single fashion. Pre-processing the features was done with Wavelet Transform and Principal Component Analysis to select the best features in the breast cancer dataset. The system correctly classified 135 out of 139 samples of the breast cancer data set with $97.12 \%$ accuracy and the preliminary results suggested that it performed better than other methods.

A proper review of the literature indicates that other systems used roulette wheel selection technique which restricted population selection. But in this paper ranked-based selection technique was adopted which does not restrict population selection. This will guarantee a higher accuracy than other reviewed papers.

\section{THEORETICAL FRAMEWORK}

The algorithm starts with a set of potential solutions (represented as a chromosome) also called a population. The solutions from one population are taken and used to form a new population. This is motivated by the hope that the new population will be better than the old one. The solutions which are selected to form new solution otherwise known as offspring are selected according to their fitness. In determining the prevalence of kidney stones, some attributes or symptoms are responded to by the users. These symptoms which include fever, vomiting, nausea, headache, etc will represent the genes in the genetic algorithm; these genes will now be combined to form a chromosome for instance a possible responder from the questionnaire is shown in table 1.

Table 1: A patient's response

\begin{tabular}{|l|l|l|l}
\hline Symptoms & \multicolumn{2}{|c|}{ Response } & Encoding \\
\hline Fever & Yes* & No & 1 \\
\hline Vomiting & Yes & No* & 0 \\
\hline Nausea & Yes* & No & 1 \\
\hline Headache & Yes* & No & 1 \\
\hline Dizziness & Yes & No* & 0 \\
\hline Stooling & Yes & No* & 0 \\
\hline Blood in the urine & Yes* & No & 1 \\
\hline Abnormal urine colour & Yes* & No & 1 \\
\hline Pain in the hips and ribs & Yes* & No & 1 \\
\hline Pains in the testicles & Yes* & No & 1 \\
\hline
\end{tabular}

The chromosome will contain information about the solution which it represents. Each chromosome has one binary string and each bit in this string can represent some characteristics of the solution. Once the chromosome has been generated, the genetic algorithm will then proceed to make crossover. The crossover selects genes from parent chromosomes and creates a new offspring. It achieves this by choosing randomly some crossover point and everything before that point is copied from a first parent and then everything after a crossover point is copied from a second parent. This is shown in table 2. After crossover, mutation takes place.

Table 2: Crossover point of the GA

Cross over point of a GA

Crossover point

\begin{tabular}{|l|l|l|}
\hline Chromosome1 & 1011 & 001111 \\
\hline Chromosome2 & 0111 & 010111 \\
\hline Offspring1 & 1011 & 010111 \\
\hline Offspring2 & 0111 & 001111 \\
\hline
\end{tabular}

The purpose of mutation is to prevent having all solutions falling into a local optimum. Mutation changes randomly the new offspring. For the binary encoding that we have, we can switch a few randomly chosen bits from 1 to 0 or from 0 to 1 . If the new generation contains a solution that produces an output that is close enough or equal to the desired answer, then the problem has been solved from our kidney stone diagnosis, the genetic algorithm assisted to avoid the pitfalls of local optima that could have occurred in the search space.

Table 3: mutation operation

\begin{tabular}{|l|l|}
\hline Original offspring1 & 101 \\
\hline Original offspring2 & 01110 \\
\hline Mutated Offspring1 & 1010010111 \\
\hline Mutated Offspring2 & 011101111 \\
\hline
\end{tabular}

The result of the genetic algorithm which is in form of a binary string (1010010111) was passed to the neural network. That is, if any of the mutated offspring proves to be the best solution from the genetic algorithm, it passes to the input layer of a feed forward neural network as shown in figure 1.

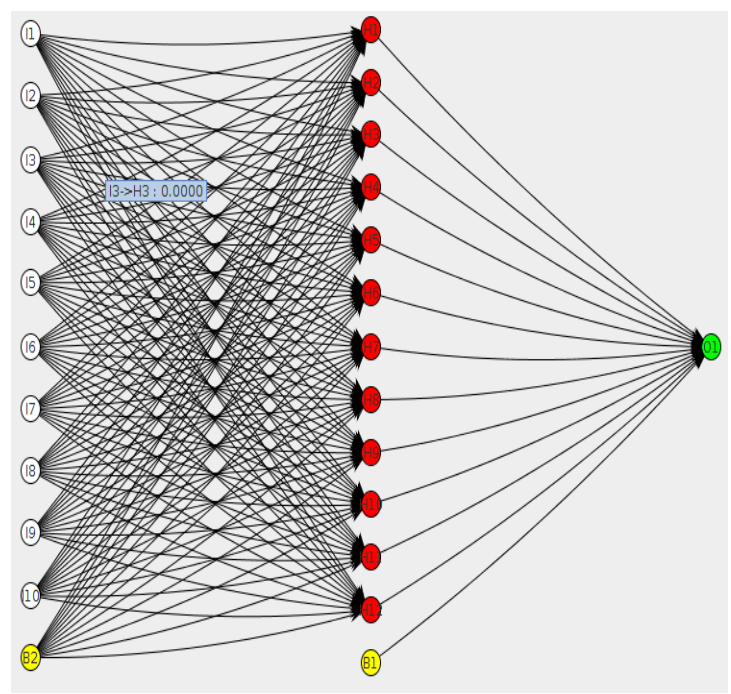

Figure 1: The Neural Network model of the system. 
Figure 1 shows the neural network model of the system which accepted chromosome generated by the genetic algorithm as input. The Network consists of ten (10) input layer denoted by the white legend, twelve (12) hidden layer denoted by the red legend and one (1) output layer denoted by the green legend. It can also be seen from the network a bias with a yellow legend attached on the input layer and hidden layer. The bias was set at the value of one to help the network effectively learn the training. The input layer accepted one neuron for each attribute of the kidney stone. The output layer outputs zero or one which stands for positive or negative.

\section{ARCHITECTURAL FRAMEWORK}

The architecture was adopted from the work of Dhanwani et al (2013) and modification made to the selection technique used in the model. The roulette wheel selection technique was replaced by the more robust and efficient ranked-based selection technique.

The ranked-based selection technique is robust and a faster selection technique which gives all the chromosomes generated a chance of being selected.

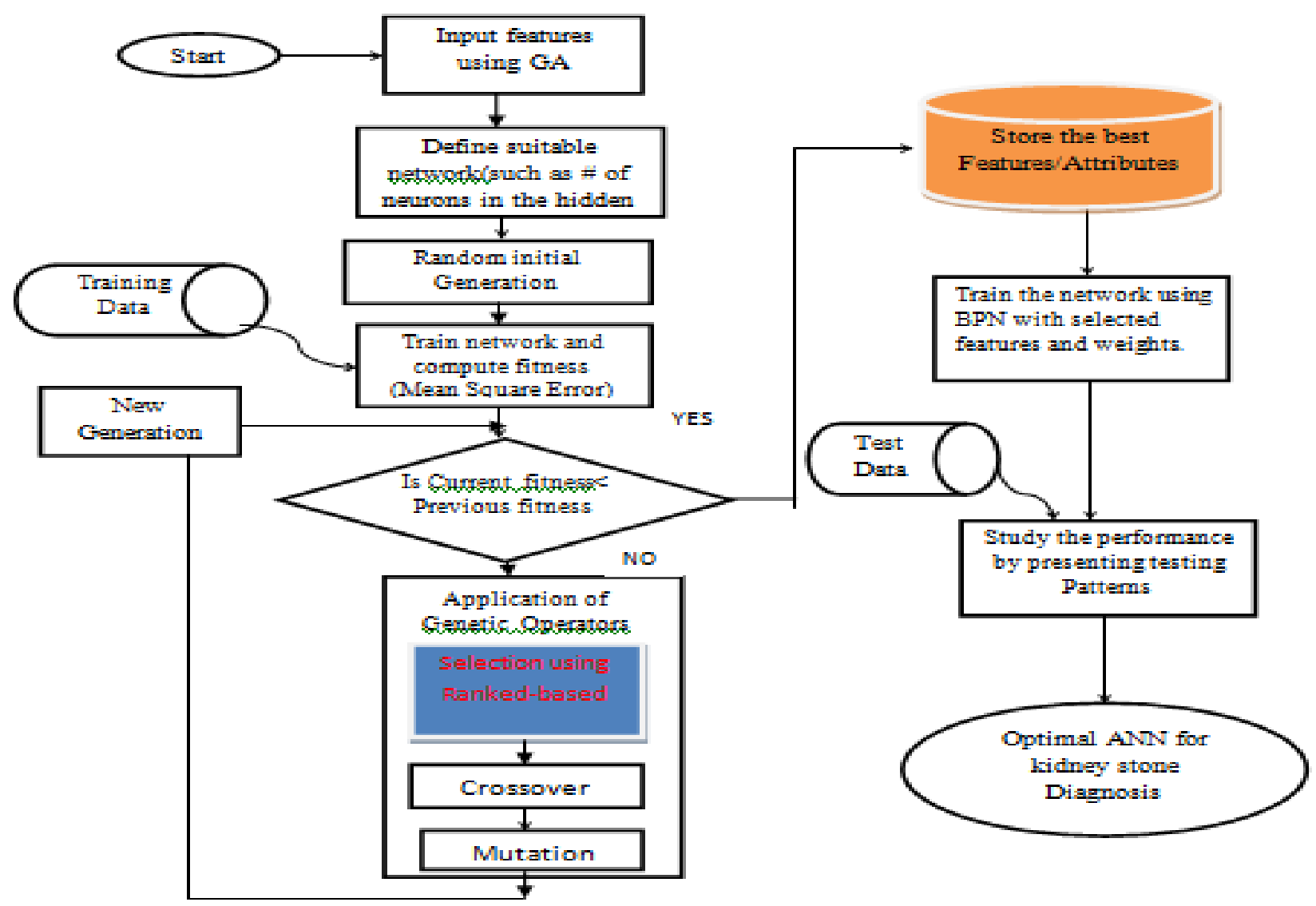

Figure 2: Architecture Diagram of the System

Figure 2 depicts the architecture of the system, the inputs interfaces with Genetic Algorithm (GA) i.e the binary numbers which represents the signs and symptoms. The number of neurons in the hidden layer is 12 neurons as shown in figure 1 which represents the signs and symptoms exhibited by the kidney stone patient.

A training set of 170 patients obtained from a hospital which is an excel document format of 28 megabyte in size was used to train the system the fitness and mean square error (MSE) is recorded. Figure 4 is the sign and symptoms interface which aid the user in interacting with the system. The response of a user in this form will produce the diagnostic report. A test data of 25 is used to test and study the performance of the system by presenting testing patterns. 


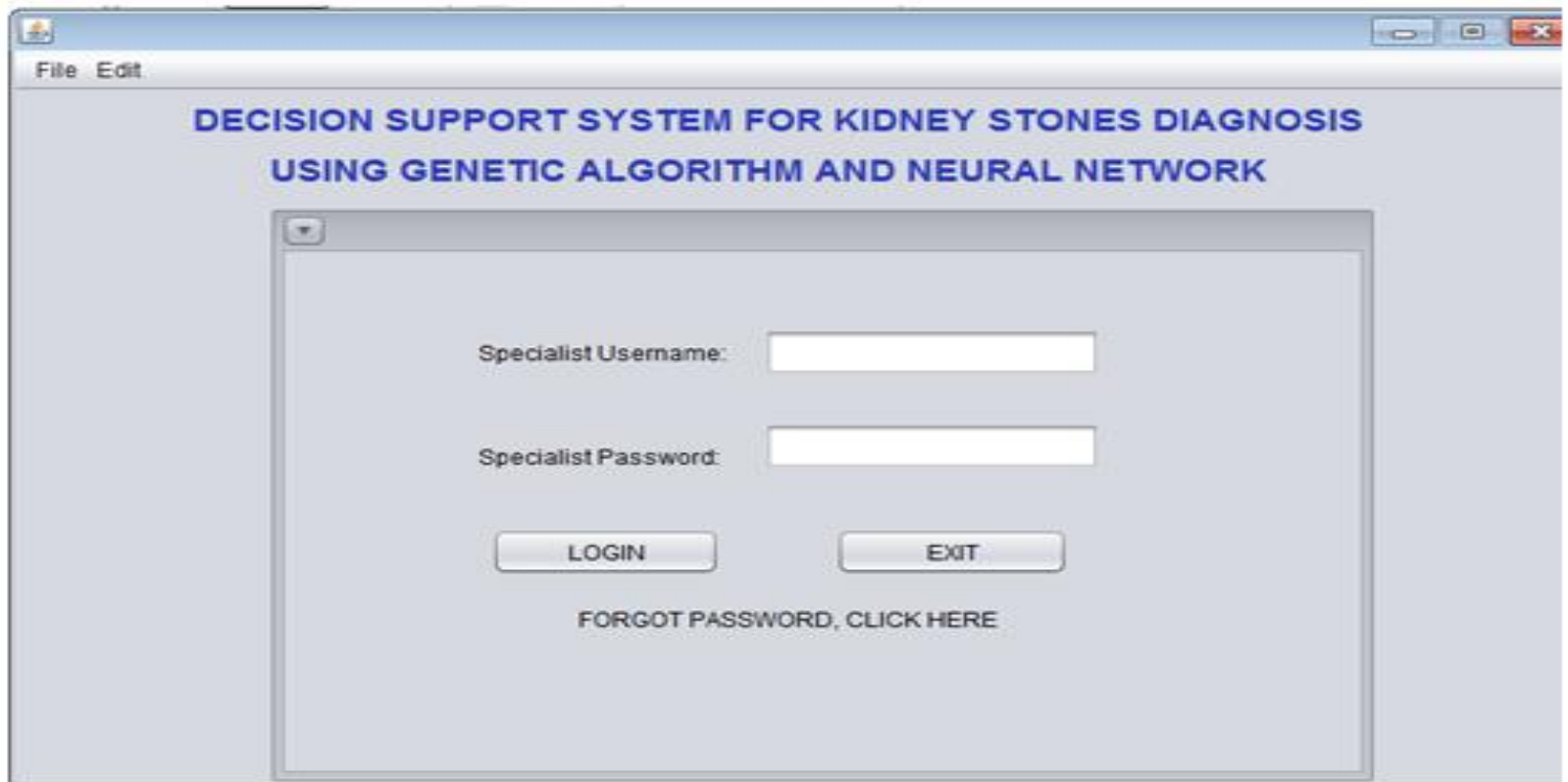

Figure 3: Login Page

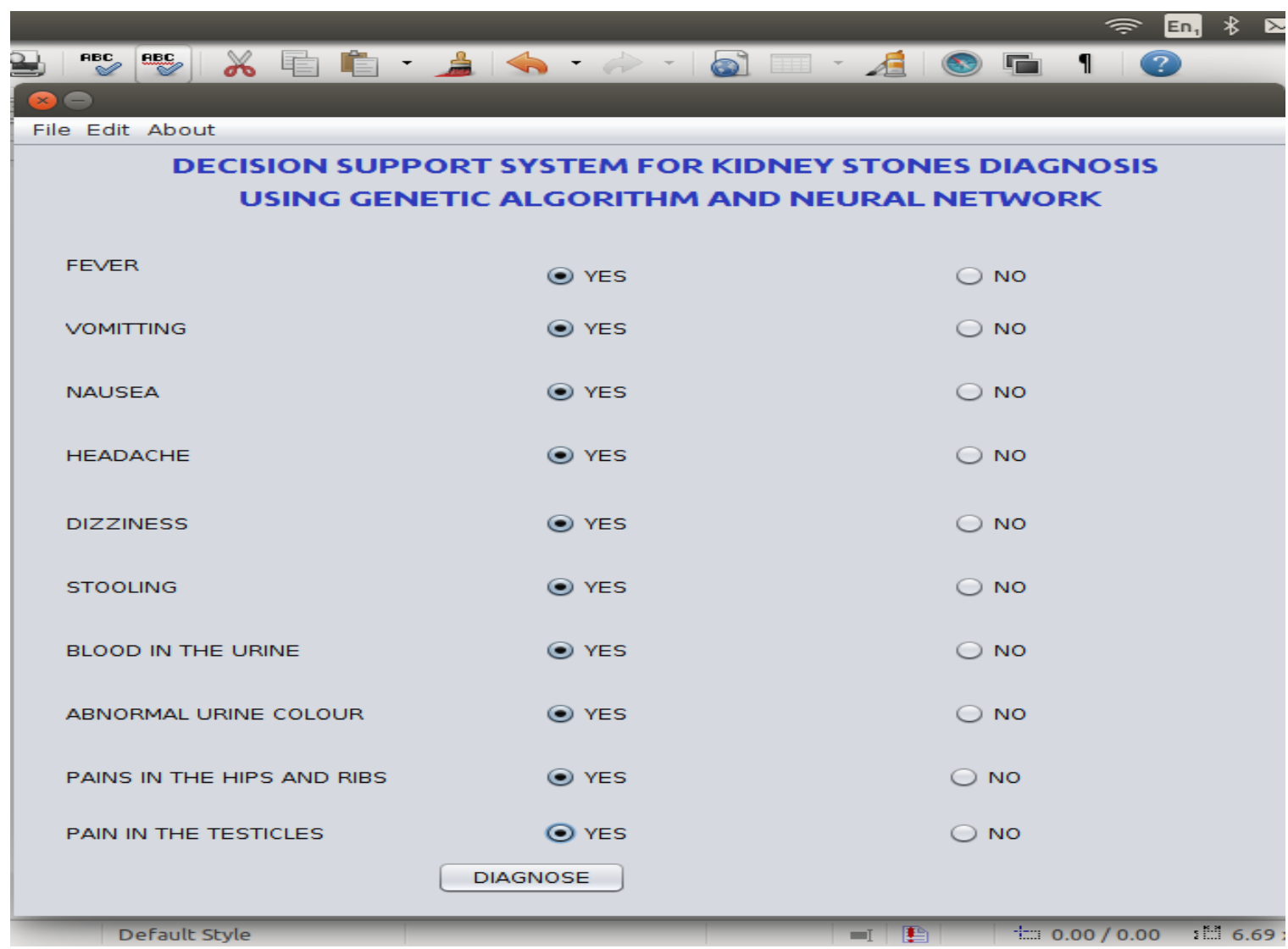

Figure 4: features interface 


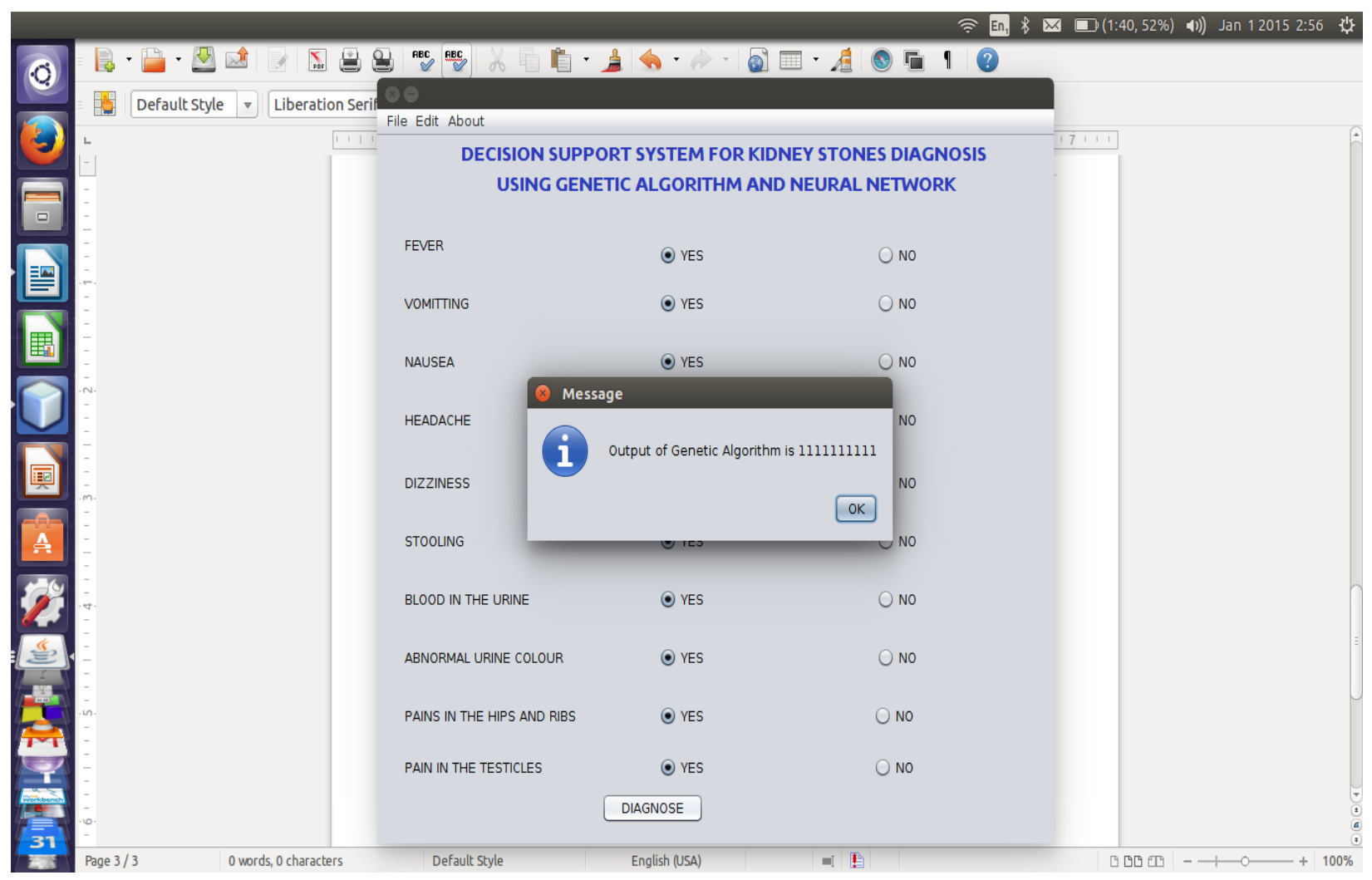

Figure 5: Output from Genetic Algorithm module

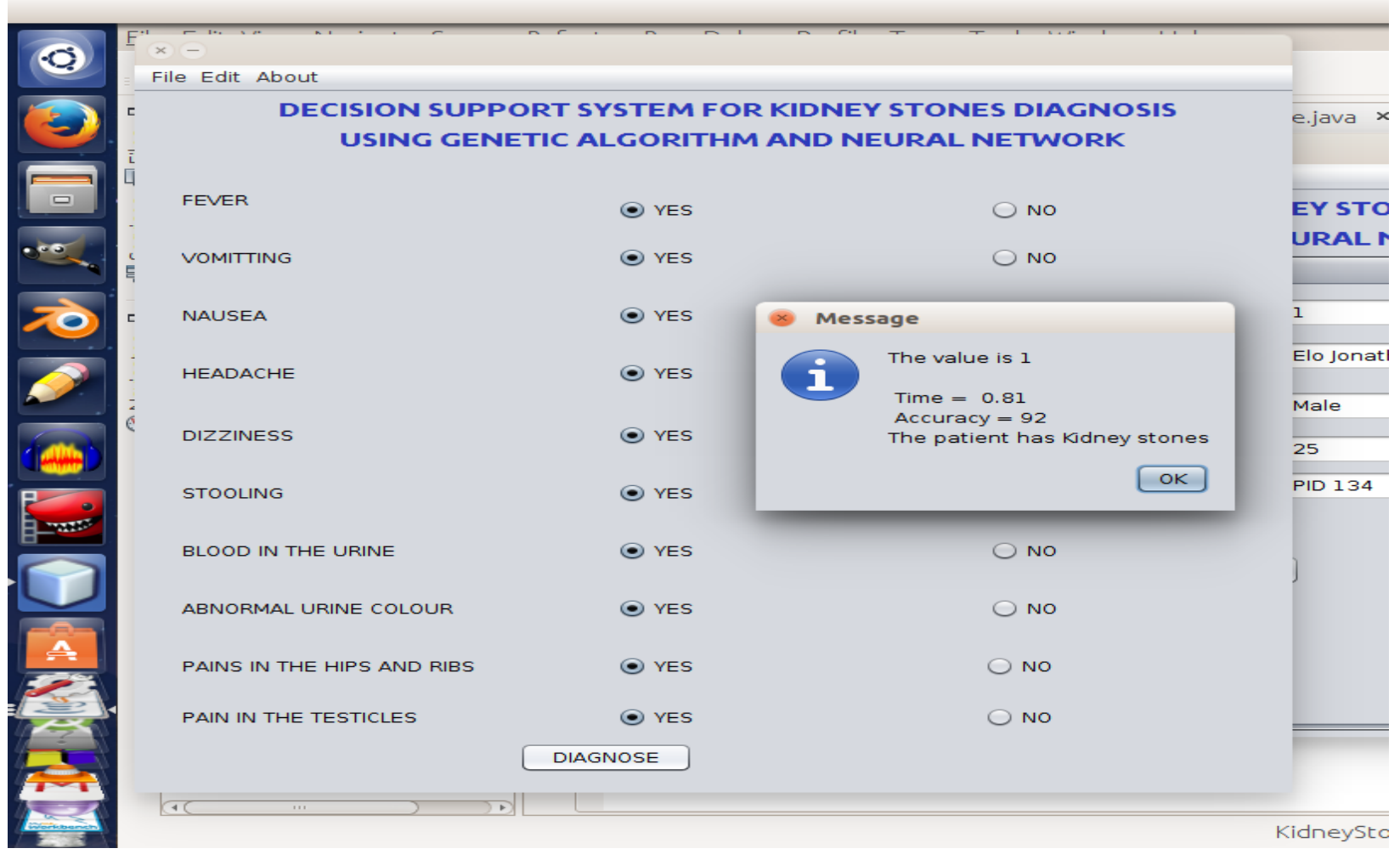

Figure 6: Report of diagnosis 


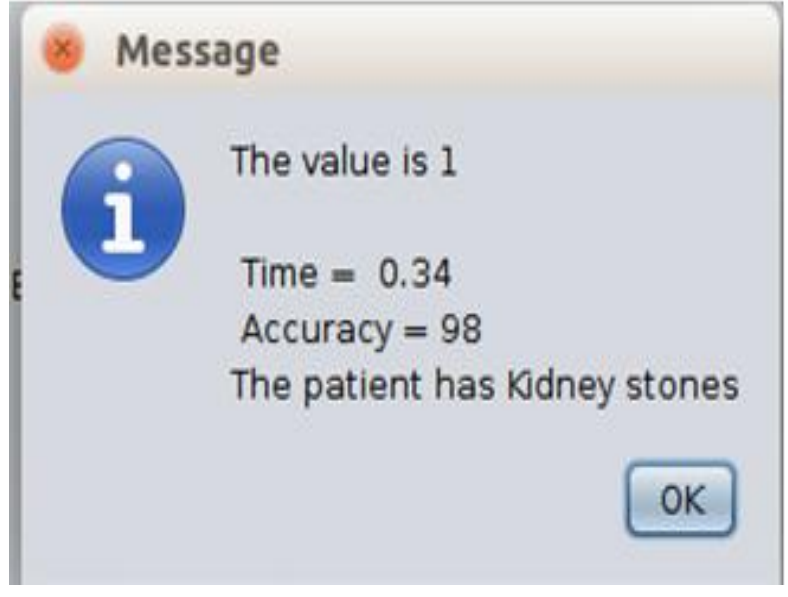

Figure 7: Report of diagnosis

Table 4: Result of Experimentations

\begin{tabular}{|c|c|c|c|}
\hline S/No & $\begin{array}{c}\text { Data Partition } \\
\text { Set }\end{array}$ & $\begin{array}{l}\text { GA-NN(based } \\
\text { on roulette } \\
\text { wheel selection } \\
\text { technique) }\end{array}$ & $\begin{array}{c}\text { GA-NN (based } \\
\text { on ranked-base } \\
\text { selection } \\
\text { technique) }\end{array}$ \\
\hline 1 & $\begin{array}{c}\text { No. of Training } \\
\text { records }\end{array}$ & 104 & 120 \\
\hline 2 & $\begin{array}{l}\text { No. of Test } \\
\text { records }\end{array}$ & 23 & 25 \\
\hline 3 & $\begin{array}{c}\text { No. of } \\
\text { Validation set }\end{array}$ & 23 & 25 \\
\hline 4 & $\begin{array}{l}\text { Feature } \\
\text { Selection } \\
\text { technique }\end{array}$ & Roulette wheel & Ranked-based \\
\hline 5 & $\begin{array}{c}\% \text { Prediction } \\
\text { accuracy }\end{array}$ & $98.67 \%$ & $98.84 \%$ \\
\hline
\end{tabular}

Table 5: Average Prediction Accuracy

\begin{tabular}{|l|l|l|l|}
\hline Approach & Training & Validation & Testing \\
\hline $\begin{array}{l}\text { Neural } \\
\text { Networks }\end{array}$ & $78.52 \%$ & $82.43 \%$ & $90.61 \%$ \\
\hline $\begin{array}{l}\text { GA-NN (based } \\
\text { on roulette } \\
\text { wheel selection } \\
\text { technique) }\end{array}$ & $79.17 \%$ & $83.88 \%$ & $98.67 \%$ \\
\hline $\begin{array}{l}\text { GA-NN (based } \\
\text { on ranked-base } \\
\text { selection } \\
\text { technique) }\end{array}$ & $80.05 \%$ & $84.25 \%$ & $98.84 \%$ \\
\hline
\end{tabular}

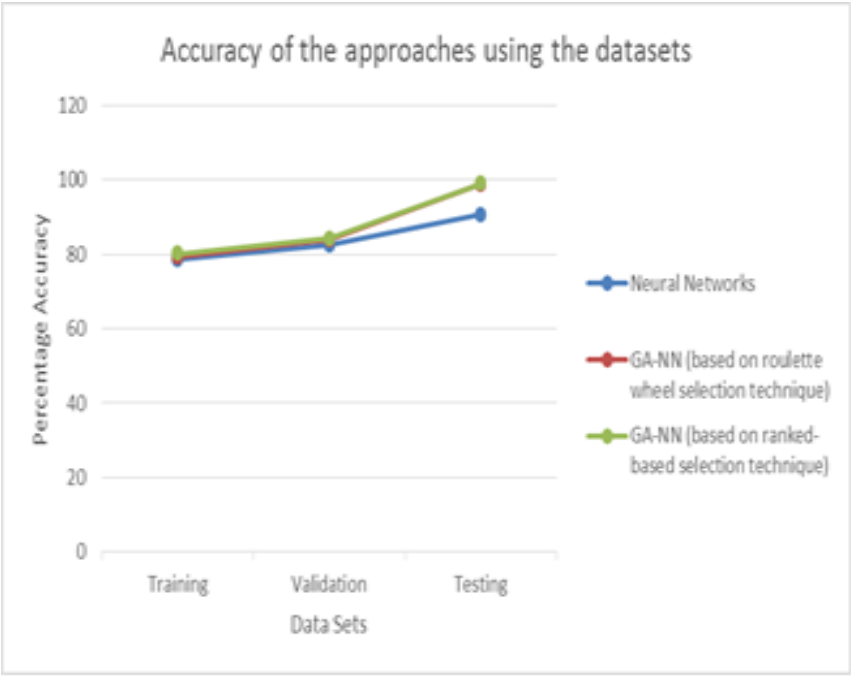

Figure 8: Level of Accuracy

\section{DISCUSSION OF RESULT}

Figures 3-7 shows interfaces for the input and output of the system respectively. Figure 3 is the login page which allows an authorized user to have access to the application. Figure 4 shows the input interface of the signs and symptoms which a patient or a user responds to for diagnosis. Figure 5 is the genetic algorithm result that was passed to neural network section of the application to produce the patients report, time of report and accuracy. Several experiments were conducted with different patients attributes to produce an average accuracy of the system. Table 4 shows the number of training set for GA-NN (based on roulette wheel selection technique) (104) and GA-NN (based on ranked-based selection technique) (120), test set (23) and (25), validation set (23) and (25) respectively. The selection techniques and the prediction accuracy of the system in percentage (\%) for GA-NN (based on roulette wheel selection technique) (98.67\%) and GA-NN (based on ranked-based selection technique) (98.84\%). Table 5 compares neural network, GA-NN (based on roulette wheel selection technique) and GA-NN (based on ranked-based selection technique) in terms of training, validation and testing as depicted in the graph in figure 8 . The result obtained with the use of the ranked-based selection technique was better than the roulette wheel; the GA-NN with ranked-based method provided better accuracy than other techniques.

\section{CONCLUSION}

A decision support system based on hybrid approach of genetic and neural network has been developed. This system can effectively be used to diagnose kidney stone and make intelligent decisions on related clinical issues. This system is not to rule out the job of the urologists, but to assist them in handling issues of clinical decision making. The experiments conducted shows that the system has very high accuracy in terms of decision making. This goes ahead to show that a hybrid system of genetic algorithm and neural network works very well in decision support system.

\section{REFERENCES}

[1] Li C, Engstrom G, and Hedblad, B. 2005. Risk factors for stroke in subjects with normal blood pressure: apropective of cohort study. Vol. 36, pp. 234-238.

[2] Sakhaee K. 2008. Nephrolithiasis as a systemic disorder.Curr opin nephrol hypertens. Vol. 17, pp. $304-$ 309 . 
[3] Amato M, Lusini ML and Nelli, F. 2004. Epidemiology of nephrolithiasis today. Urol int. Vol.72, pp. 1-5.

[4] Robertson WG, Peacock M and Baker, M. 1983 studies on the prevalence and epidemiology of urinary stone Disease in men in leeds. Br jUrol, Vol. 55, pp. 595-598.

[5] Cirillo M and Laurenzi, M. 1998. Elevated blood pressureand positive history of kidney stones: Results from apopulation-based study. J hypertens suppl. Vol. 6, pp. $485-486$

[6] Leonetti F, Dussol B and Berthezene, P. 1998. Dietary andurinary risk factors for stones in idiopathic calciumstone formers compared with healthy subjects. Nephrol Dial transplant. Vol 13, pp. 617-622.

[7] Curhan G.C, Willett W.C and Rimm, E.B. 1998 Body size and risk of kidney stones. J Am soc Nephrol. Vol. 9, pp. $1645-1652$

[8] Clinical Applications of Artificial Neural NetworksEdited by Richard Dybowski King's College London Edited byVanya Gant University CollegeLondon Hospitals NHS Trust, London. 2007.

[9] Dybowski R. and Gant, V. 2007 ClinicalApplications of Artificial Neural Networks, Cambridge University Press.

[10] Moein, S Monadjemi S.A and Moallem, P 2009A NovelFuzzy-Neural Based Medical DiagnosisSystem, International Journal of Biological \&Medical Sciences, Vol. 4(3), pp. 146-150.

[11] Saigal C.S, Joyce G and Timilsina, A.R. 2005. Urologicdiseases in America project. Direct and indirectcosts of nephrolithiasis in an employed population: opportunity for disease management? Kidney intVol. 5, pp. 1808-1814.

[12] Chiang D, Chiang, H.C, Chen W.C and Tsai, F.J. 2002Prediction of stone disease by Discriminant analysis and artificial neural networks in genetic polymorphisms: a new method, B J U InternationalVol. 9(1), pp. 661-666.

[13] Bäck T, F. Hoffmeister, and Schwefel, H.P. 1991 Asurvey of evolutionary strategies. In R. Belew andL. Booker, editors, Prooceedings of the FourthInternational Conference on GeneticAlgorithms, pp. 2-9, San Mateo, $\mathrm{CA}$

[14] Irfan Y. Khan, P.H. Zope, Suralkar, S.R. 2013Importance of Artificial Neural Network in MedicalDiagnosis disease like acute nephritis disease andheart disease, International Journal of EngineeringScience and Innovative Technology (IJESIT), Vol. 2(2), pp. 210-217.
[15] Bryan A Liang, 1999. Management and prevention ofNephrolithiasis: outcomes-based practice; HospitalPhysician Vol. 2, pp. 22-37

[16] Lisboa, P. J.2002. A Review of Evidence of HealthBenefit from Artificial Neural Networks in MedicalIntervention. Neural Networks, Vol. 15(1), pp. $11-39$.

[17] Dhanwani, Deepak and Avinash, 2013. Study ofHybrid Genetic Algorithm Using Artificial Neural Network in Data Mining for the Diagnosis of Stroke Disease, International journal of Computational Engineering Research. Vol. 3(4), pp. 95-100.

[18] Abhishek, Gour S.M.T and Dolly, G. 2012 ProposingEfficient Neural Network Training Model for kidneyStone Diagnosis, International journal of ComputerScience and Information Technologies, Vol 3(3),pp.3900-3900.

[19] Koshal K, Kumar and Abhishek, 2012. Artificial NeuralNetworks for Diagnosis of kidney stones disease, International journal of information Technology andComputer Science. Vol. 7, pp. 20-25.

[20] Qeethara K.A. 2011. Artificial Neural Network inMedical Diagnosis, International journal of ComputerScience issues. Vol. 8(2); 150-154.

[21] Hamada R.H, Al-Absi, Azween Abdullah, Mahama IsaaHassan, and Khaled Bashir shaban. 2011. HybridIntelligent System for Disease Diagnosis Based onArtificial Neural Network, Fuzzy Logic, and GeneticAlgorithms, ICIEIS, Part II, CCIS. Vol. 252, pp. 128-139, Springer-Verlag Berlin Heidelberg.

[22] Sneha, A.M and chougule, S.R 2014. A Review on Neural Network for Diagnosis of KidneyStone, International journal of Science andResearch. ISSN (online): 2319-7064.

\section{AUTHOR PROFILE}

EMETO, Ifeanyi Charles received a Bachelor of Science (B.Sc.) degree in Computer Science from University of Port Harcourt, Nigeria and a Master of Science (M.Sc.) degree from the same University. His research interest is in the area of Machine Learning.

Ugwu, Chidiebere is a Senior Lecturer in the Department of Computer Science University of Port Harcourt. His research interest is Machine learning, Modeling and Simulation and Algorithm. He has published numerous papers detailing his research findings both in local and International Journals. He is a reviewer for many local and International journals. He is also a member of many Professional bodies including NCS, IEEE, CPN, IRDI. 